\title{
EXPERIMENTAL IMPLEMENTATION OF INTEGRATOR BACKSTEPPING AND PASSIVE NONLINEAR CONTROLLERS ON THE RTAC TESTBED
}

\author{
ROBERT T. BUPP ${ }^{1}$, DENNIS S. BERNSTEIN ${ }^{2 *}$ AND VINCENT T. COPPOLA ${ }^{2}$ \\ ${ }^{1}$ TRW, One Space Park, Redondo Beach, CA 90278, U.S.A. \\ ${ }^{2}$ Department of Aerospace Engineering, The University of Michigan, Ann Arbor, MI 48109-2140, U.S.A.
}

\begin{abstract}
SUMMARY
This paper fully describes the RTAC experimental testbed which provides a means for implementing and evaluating nonlinear controllers. The description of the testbed includes all physical parameters of the device that are relevant to the design and implementation of controllers. Next, four nonlinear controllers are considered. The first controller is a static, full-state-feedback, globally asymptotically stabilizing control law developed using partial feedback linearization and integrator backstepping. Next, three nonlinear controllers based upon passivity principles are presented, two of which are encompassed by the classical passivity framework, while the third is based upon the novel concept of virtual resetting absorbers. The passive controllers do not require either translational position or velocity measurements. All of the controllers are implemented on the RTAC testbed and their performance is examined. (C) 1998 John Wiley \& Sons, Ltd.

Key words: Rotational/translational actuator (RTAC); passivity; dissipative control; virtual resetting absorbers; integrator backstepping
\end{abstract}

\section{INTRODUCTION}

The rotational/translational actuator (RTAC) provides a low-dimensional nonlinear system for investigating nonlinear control techniques. ${ }^{1-5}$ The lossless formulation of this problem involves the nonlinear coupling of an undamped oscillator with a rotational rigid body mode. Stabilization and disturbance rejection objectives for this problem have been formulated as a benchmark problem. ${ }^{4}$

This paper has three objectives. First, the RTAC experimental testbed is fully described. This testbed has been designed to provide the means for implementing and evaluating a variety of nonlinear controllers. The description of the RTAC testbed includes all physical parameters of the device that are relevant to the design and implementation of controllers. Next, we consider several nonlinear control designs for the RTAC, including integrator backstepping and passivitybased controller designs. Finally, all of the controllers are implemented on the RTAC testbed and their performance is evaluated.

The integrator backstepping design is based on the work of Wan et al. ${ }^{1,2}$ This approach requires that the equations of motion be reformulated by partial feedback linearization. ${ }^{6}$

\footnotetext{
* Correspondence to: Dennis S. Bernstein, Department of Aerospace Engineering, University of Michigan, 1320 Beal Street, Ann Arbor, MI 48109-2140, U.S.A.
}

CCC 1049-8923/98/050435-23\$17.50

(C) 1998 John Wiley \& Sons, Ltd. 
Integrator backstepping ${ }^{7}$ is then used to produce a family of globally asymptotically stabilizing control laws.

Three passivity-based controllers are developed for the RTAC. These controllers have intuitively appealing energy-dissipative properties and thus also inherent stability robustness to plant and disturbance uncertainty. Two of these controllers are encompassed by the classical passivity framework, ${ }^{8-14}$ with versions of these controllers appearing in References 3 and 4 . The final controller is based upon the novel concept of resetting absorbers. ${ }^{15,16}$

\section{EXPERIMENTAL TESTBED}

An RTAC experimental testbed has been constructed based on the nonlinear system model of References 1-4 to evaluate the performance of various nonlinear controllers. A photograph of the testbed appears in Figure 1. A model of this arrangement, illustrated by Figure 2, consists of a translational cart of mass $M$ connected by a spring of stiffness $k$ to a wall. The rotational actuator, which is mounted on the cart, consists of a proof mass of mass $m$ and centroidal moment of inertia $I$ mounted at a fixed distance $e$ from its centre of rotation. A control torque denoted by $N$ is applied to the rotational proof mass.

Let $q$ denote the translational position of the cart from its equilibrium position, and let $\theta$ denote the counterclockwise rotational angle of the eccentric mass, where $\theta=0$ is perpendicular to the direction of translation, as shown in Figure 2. The equations of motion are given by

$$
\begin{aligned}
(M+m) \ddot{q}+k q & =-m e\left(\ddot{\theta} \cos \theta-\dot{\theta}^{2} \sin \theta\right) \\
\left(I+m e^{2}\right) \ddot{\theta} & =-m e \ddot{q} \cos \theta+N
\end{aligned}
$$

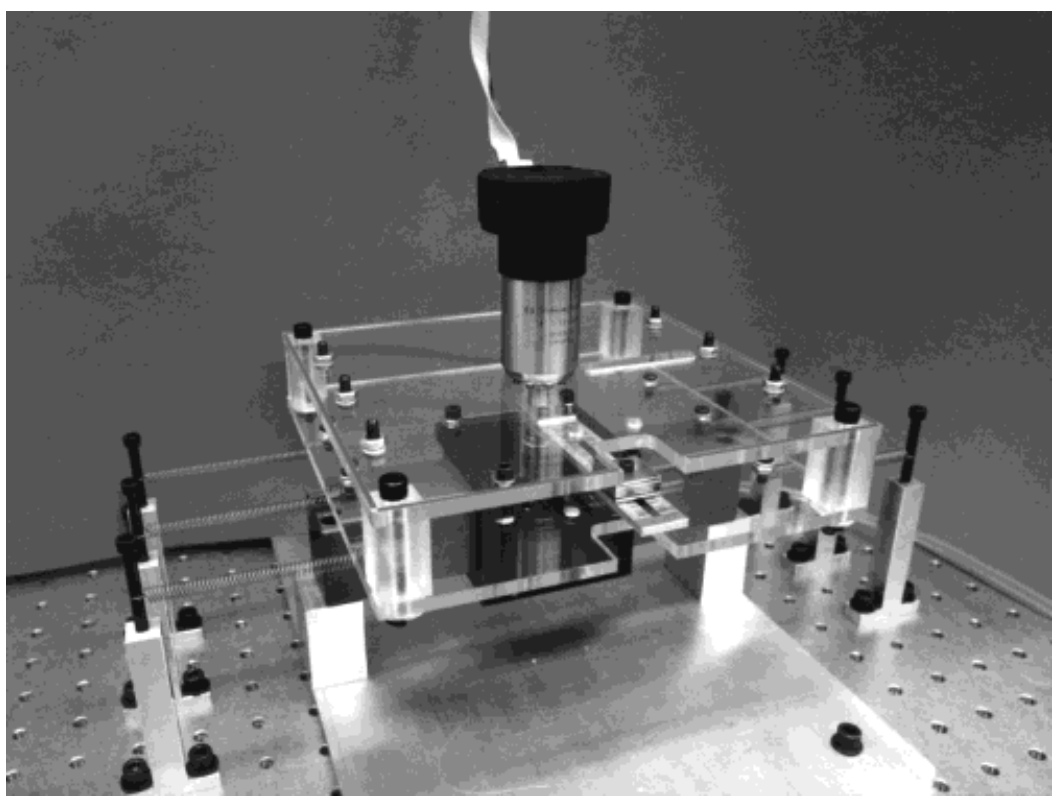

Figure 1. The RTAC testbed 


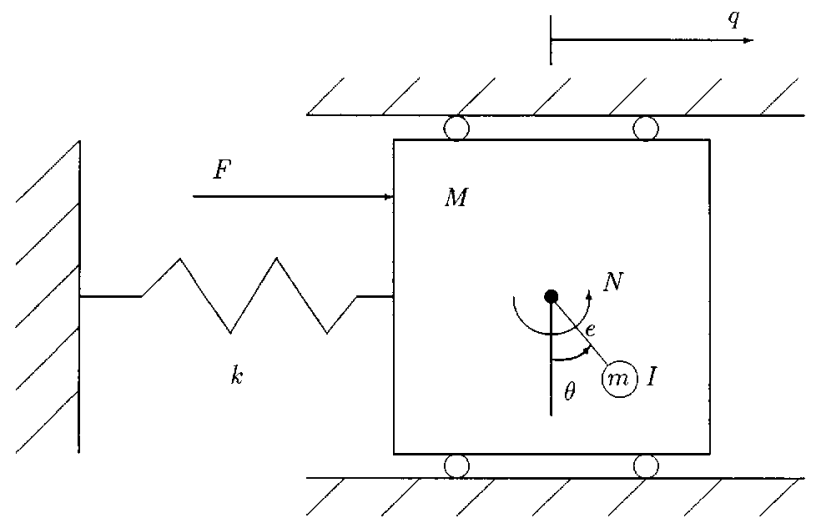

Figure 2. Model of the RTAC experiment

Note that while the model is lossless, the experimental testbed experiences unmodelled damping effects such as air and motor friction.

Equations (1) and (2) are given in first-order form by

$$
\dot{x}=f(x)+g(x) N
$$

where $x=[q, \dot{q}, \theta, \dot{\theta}]^{\mathrm{T}}$,

$$
f(x)=\left[\begin{array}{c}
\dot{q} \\
\frac{-\left(I+m e^{2}\right)\left(k q-m \dot{\theta}^{2} \sin \theta\right)}{(M+m)\left(I+m e^{2}\right)-(m e \cos \theta)^{2}} \\
\dot{\theta} \\
\frac{m e \cos \theta\left(k q-m \dot{\theta}^{2} \sin \theta\right)}{(M+m)\left(I+m e^{2}\right)-(m e \cos \theta)^{2}}
\end{array}\right], \quad g(x)=\left[\begin{array}{c}
0 \\
\frac{-m e \cos \theta}{(M+m)\left(I+m e^{2}\right)-(m e \cos \theta)^{2}} \\
0 \\
M+m \\
\frac{M+m)\left(I+m e^{2}\right)-(m e \cos \theta)^{2}}{(M+m}
\end{array}\right]
$$

The translational cart consists of a pair of acrylic plates together with some mounting hardware. The spring $k$ is realized by $2-12$ helical springs mounted symmetrically and pretensioned. The control torque $N$ is implemented by means of a rotary DC brush motor, onto whose shaft is mounted an eccentric arm. The cart is mounted on an air bearing, resulting in low-friction, one-dimensional motion. The remainder of this section provides a more detailed description of the experimental setup, including hardware components and controller implementation.

The translational cart is constructed of two $8^{\prime \prime} \times 8^{\prime \prime} \times 0 \cdot 25^{\prime \prime}$ acrylic plates. The lower plate is bolted to a $3^{\prime \prime} \times 3^{\prime \prime}$ carriage, which is constrained to ride on the air slide. The air slide has a usable length of $7^{\prime \prime}$, thus allowing \pm 2 " of travel from the centre. The upper plate is mounted directly above the lower plate on $1 \cdot 125^{\prime \prime}$ tall acrylic mounting posts located at the corners of the plates. The DC motor is mounted on the upper acrylic plate with its shaft pointing downward. The springs have an experimentally measured stiffness of $3 \cdot 1 \mathrm{oz} / \mathrm{in}$, and the nominal configuration of six springs attached yields an effective spring constant of $k=18.6 \mathrm{oz} / \mathrm{in}$.

With the arm motionless at $90^{\circ}$ there is no coupling between the translational and rotational motions. By measuring the experimental free response, the natural frequency of the oscillator in 
this configuration was determined to be $1.64 \mathrm{~Hz}$. Since the way in which the experiment was constructed made it difficult to determine the mass of each subpart precisely, the measured stiffness and the measured natural frequency were used to identify the effective mass of the cart. This total mass $M$ consisting of the masses of the acrylic plates, the mounting section of the air slide, the DC motor, and the mounting hardware was determined to be approximately $65 \mathrm{oz}$. The damping ratio of the oscillator with the eccentric arm held motionless was also determined from the free response characteristics and was seen to vary from approximately $0.45 \%$ when the RTAC is configured for experimentation, to less than $0 \cdot 1 \%$ when no power or data cables are attached. The supply hose for the air slide must remain attached to the cart, however, and the air drag resulting from the motion of the hose and the cart is responsible for much of the remaining damping.

The eccentric arm connected to the motor shaft is a $4 \cdot 25^{\prime \prime} \times 0 \cdot 75^{\prime \prime} \times 0 \cdot 0625^{\prime \prime}$ aluminium bar, into which a $2 \cdot 75^{\prime \prime}$ long slot is cut to facilitate mounting additional proof masses. The bar is attached to a steel collar which is fastened with set screws to the shaft of the DC motor. While the eccentric arm alone serves as a rotational proof mass, additional proof masses constructed of steel can be mounted to the eccentric arm at a range of locations. The additional proof masses are each $0.75^{\prime \prime} \times 0.75^{\prime \prime} \times 0.0625^{\prime \prime}$ and weigh $0.14 \mathrm{oz}$. They can be stacked as many as six high, and mounted on the arm between $1 \cdot 5^{\prime \prime}$ and $3 \cdot 25^{\prime \prime}$ from the motor shaft. The aluminium arm is scored at $0 \cdot 125^{\prime \prime}$ intervals to permit repeatability of the experimental configuration when additional proof masses are attached. The effective values of the parameters $m, e$, and $I$ vary depending on the number and location of the attached proof masses. With no proof masses attached to the arm, $m=1.56 \mathrm{oz}$, $e=1.76^{\prime \prime}$ and $I=0.74 \mathrm{oz} \mathrm{in}^{2}$, where $m$ is measured, and $e$ and $I$ are calculated based on geometry and material properties. When no additional proof masses are attached to the arm, the value of the coupling parameter $\varepsilon$, which will be defined in Section 3 , is $\varepsilon=0 \cdot 14$. In a typical configuration of 5 additional proof masses mounted on the arm $3 \cdot 875^{\prime \prime}$ from the rotation axis, the coupling increases to $\varepsilon=0 \cdot 18$. The physical parameters of the RTAC testbed are summarized in Table I.

The torque actuation is provided by a $12 \mathrm{~V} \mathrm{DC}$ brush motor which is $2 \cdot 25^{\prime \prime}$ long and $1 \cdot 38^{\prime \prime}$ in diameter. The motor produces $3.04 \mathrm{oz}$ in/A, and is rated for continuous torque of $14.2 \mathrm{oz}$ in, and continuous power output of $45 \mathrm{~W}$. To achieve the torque ratings, the motor is capable of operating with a continuous 4.67 A load, although higher currents can be tolerated for short periods of time. In order to utilize torque as the control input, we use a DC servo drive to command the current into the motor. The pulse-width-modulated drive is rated to produce $5 \mathrm{~A}$ continuous current and 10 A peak current with a bandwidth of $2.5 \mathrm{kHz}$.

Table I. Summary of the RTAC physical parameters

\begin{tabular}{llcc}
\hline & & Bare arm & $\begin{array}{c}5 \text { Added proof } \\
\text { masses }\end{array}$ \\
\hline Cart mass & $M$ & $65.5 \mathrm{oz}$ & $65.5 \mathrm{oz}$ \\
Arm mass & $m$ & $1.56 \mathrm{oz}$ & $2.28 \mathrm{oz}$ \\
Spring constant & $k$ & $18.6 \mathrm{oz} / \mathrm{in}$ & $18.6 \mathrm{oz} / \mathrm{in}$ \\
Eccentricity & $e$ & $1.76 \mathrm{in}$ & $2 \cdot 43 \mathrm{in}$ \\
Arm inertia & $I$ & $0.74 \mathrm{oz}$ in $^{2}$ & $0.74 \mathrm{oz}$ in \\
Coupling & $\varepsilon$ & 0.14 & $0 \cdot 18$ \\
\hline
\end{tabular}


To avoid damaging the motor, a number of mechanisms are utilized to ensure that the current into the motor is limited. In the hardware elements of the control system, fuses of various ratings can be installed. A 4 A, fast-blow-type fuse is typically used. Software-based current limiting is also implemented. Since the servo drive is configured for a transconductance of $0.5 \mathrm{~A}$ output per unit voltage command, we limit the current command to the motor by inserting a software voltage saturation element in the controller. When a controller is first implemented, the software saturation may be set fairly low, whereas for well-tested controllers the software limiting is usually set to $\pm 8 \mathrm{~V}$, limiting the current command to $\pm 4 \mathrm{~A}$. Note that the software limiting does not have the same effect as the hardware limiting, since reaching a software limit entails control signal saturation, whereas blowing a fuse immediately disconnects the control.

The angular position of the eccentric arm is sensed by an incremental optical encoder mounted on the motor. The encoder has 1000 lines per revolution and two-channel quadrature output ( 4 'edges' per encoder line) yielding an effective angular resolution of $360^{\circ} / 4000=0.09^{\circ}$. Due to limitations in the encoder electronics the maximum angular rate of the arm for which the encoder will be effective is $1250 \mathrm{rad} / \mathrm{s}$, well above the bandwidth of the system. To estimate angular velocity, we apply a finite difference scheme to the angular position data.

Translational displacement of the cart is measured by a linear variable displacement transducer (LVDT). The LVDT consists of an $5 \cdot 3^{\prime \prime}$ long stationary body that is mounted to the table and a $11 \cdot 1^{\prime \prime}$ long core that is attached to the cart. The core rides inside the body of the LVDT without contact. The LVDT has an output range of \pm 2 " and an output gain of 5.0 V per inch, producing a $\pm 10 \mathrm{~V}$ maximum output. The output is linear to within $25 \mathrm{mV}$, or $0.005 \mathrm{in}$. To estimate translational velocity, we apply a finite difference scheme to the translational position data.

To implement real-time control, we use a dSPACE ds1102 control board in conjunction with the Real Time Workshop software which provides an interface to Matlab's Simulink. As a result, controllers that can be represented by Simulink block diagrams without function calls to external M-files can be automatically converted to assembly code for implementation on the control board. This arrangement allows for parameterized control laws, where the parameters can be displayed and adjusted while the experiment is running.

The control board has two 12-bit A/D inputs, two 16-bit A/D inputs, two encoder inputs, and four 12-bit D/A output channels, as well as digital I/O channels. The input from the LVDT is sampled by a 16-bit A/D converter, and the command to the servo drive is sent to a 12-bit D/A channel. The control board, which is resident in a $90 \mathrm{MHz}$ Pentium-based host PC, utilizes an internal $40 \mathrm{MHz}$ Texas Instruments TMS320C31 floating point digital signal processor for control. Sample rates for the controllers considered in this paper are $5 \mathrm{kHz}$, corresponding to a $200 \mu$ s sampling period.

\section{INTEGRATOR BACKSTEPPING CONTROLLER}

In this section we review the integrator backstepping design procedure used in Reference 2 to obtain a family of globally asymptotically stabilizing controllers for (3). Following the procedure in Reference 2, we normalize the equations of motion, scaling displacement, torque, and time according to

$$
\xi \triangleq \sqrt{\frac{M+m}{I+m e^{2}}} q, \quad v \triangleq \sqrt{\frac{M+m}{k\left(I+m e^{2}\right)}} N, \quad \tau \triangleq \sqrt{\frac{k}{M+m}} t
$$


to obtain

$$
\begin{aligned}
\ddot{\xi}+\xi & =\varepsilon\left(\dot{\theta}^{2} \sin \theta-\ddot{\theta} \cos \theta\right) \\
\ddot{\theta} & =-\varepsilon \ddot{\xi} \cos \theta+v
\end{aligned}
$$

In the normalized equations (5)-(6) the parameter $\varepsilon$ defined as

$$
\varepsilon \triangleq \frac{m e}{\sqrt{\left(I+m e^{2}\right)(M+m)}}
$$

quantifies the coupling between the translational and rotational motions. Note that in this section the notation ( ${ }^{\circ}$, when applied to the scaled variables, denotes differentiation with respect to the scaled time $\tau$.

We next apply a change of co-ordinates motivated by the geometric properties of (3). The diffeomorphic transformation that achieves partial feedback linearization is given by

$$
z_{1} \triangleq \xi+\varepsilon \sin \theta, \quad z_{2}=\triangleq \dot{\xi}+\varepsilon \dot{\theta} \cos \theta, \quad y_{1} \triangleq \theta, \quad y_{2} \triangleq \dot{\theta}
$$

Equation (3) can now be rewritten in terms of the variables $z_{1}, z_{2}, y_{1}$ and $y_{2}$ as

$$
\begin{aligned}
& \dot{z}_{1}=z_{2} \\
& \dot{z}_{2}=-z_{1}+\varepsilon \sin y_{1} \\
& \dot{y}_{1}=y_{2} \\
& \dot{y}_{2}=v
\end{aligned}
$$

where

$$
v=\frac{\varepsilon \cos y_{1}}{1-\varepsilon^{2} \cos ^{2} y_{1}}\left[z_{1}-\left(1+y_{2}^{2}\right) \varepsilon \sin y_{1}\right]+\frac{1}{1-\varepsilon^{2} \cos ^{2} y_{1}} v
$$

The integrator backstepping procedure [7] can now be used to develop a globally asymptotically stabilizing control law in terms of the variable $v$. To do this, we find a function $y_{1}\left(z_{1}, z_{2}\right)$ that globally asymptotically stabilizes (8), (9), and then backstep twice to obtain a control $v$ that globally asymptotically stabilizes (8)-(11). The transformation (12) is used to determine the corresponding scaled torque $v$, and the scaling (4) is used to determine the actual torque command $N$. In terms of the scaled variables $\xi, \theta, \tau$ and $v$, the control law derived in Reference 2 is given by

$$
\begin{aligned}
v= & {\left[-\left(c_{1}+c_{2}\right) \dot{\theta}-\left(c_{1} c_{2}+\frac{p_{1}}{p_{2}}\right)\left(\theta+c_{0} \arctan (\dot{\xi}+\varepsilon \dot{\theta} \cos \theta)\right)+\frac{c_{0}\left(\left(c_{1}+c_{2}\right) \xi+\dot{\xi}\right)}{1+(\dot{\xi}+\varepsilon \dot{\theta} \cos \theta)^{2}}\right.} \\
& -\frac{\varepsilon p_{0}\left(c_{2} \dot{\xi}+c_{2} \varepsilon \dot{\theta} \cos \theta-\xi\right)\left[\sin \theta+\sin \left(c_{0} \arctan (\dot{\xi}+\varepsilon \dot{\theta} \cos \theta)\right)\right]}{p_{1}\left(\theta+c_{0} \arctan (\dot{\xi}+\varepsilon \dot{\theta} \cos \theta)\right)}
\end{aligned}
$$




$$
\begin{aligned}
& +2 \frac{c_{0} \xi^{2}(\dot{\xi}+\varepsilon \dot{\theta} \cos \theta)}{\left(1+(\dot{\xi}+\varepsilon \dot{\theta} \cos \theta)^{2}\right)^{2}}+\frac{\varepsilon p_{0} c_{0} \xi(\dot{\xi}+\varepsilon \dot{\theta} \cos \theta) \cos \left(c_{0} \arctan (\dot{\xi}+\varepsilon \dot{\theta} \cos \theta)\right)}{p_{1}\left(1+(\dot{\xi}+\varepsilon \dot{\theta} \cos \theta)^{2}\right)\left(\theta+c_{0} \arctan (\dot{\xi}+\varepsilon \dot{\theta} \cos \theta)\right)} \\
& -\frac{\varepsilon p_{0}(\dot{\xi}+\varepsilon \dot{\theta} \cos \theta) \dot{\theta} \cos \theta}{p_{1}\left(\theta+c_{0} \arctan (\dot{\xi}+\varepsilon \dot{\theta} \cos \theta)\right)}+\frac{\varepsilon p_{0} \dot{\theta}(\dot{\xi}+\varepsilon \dot{\theta} \cos \theta)\left[\sin \theta+\sin \left(c_{0} \arctan (\dot{\xi}+\varepsilon \dot{\theta} \cos \theta)\right)\right]}{p_{1}\left(\theta+c_{0} \arctan (\dot{\xi}+\varepsilon \dot{\theta} \cos \theta)\right)^{2}} \\
& \left.-\frac{\varepsilon \cos \theta}{1-\varepsilon^{2} \cos ^{2} \theta}\left[\xi-\dot{\theta}^{2} \varepsilon \sin \theta\right]\right]\left(1-\varepsilon^{2} \cos ^{2} \theta\right)
\end{aligned}
$$

where the controller parameters $p_{0}, p_{1}, p_{2}, c_{0}, c_{1}$ and $c_{2}$ are all positive, and $0<c_{0}<2$. Global asymptotic stability of the origin is obtained for every allowable choice of these parameters.

\section{PASSIVE SYSTEMS}

In this section, we define dissipativity, passivity, and losslessness for a general class of dynamical systems. We then restrict our attention to the control systems considered in this paper, and give sufficient conditions that guarantee the passivity of these systems.

A dynamical system with input $u(t) \in U \subseteq \mathbb{R}^{m}$, output $y(t) \in Y \subseteq \mathbb{R}^{p}$, and internal state $x(t) \in X \subseteq \mathbb{R}^{n}$ is said to be dissipative if there exists a continuous storage function $V_{s}: X \rightarrow[0, \infty)$ and a supply rate $w: U \times Y \rightarrow \mathbb{R}$ such that, for all $t>0$, the dissipation inequality

$$
V_{s}(x(t))-V_{s}(x(0)) \leqslant \int_{0}^{t} w(u(s), y(s)) \mathrm{d} s
$$

is satisfied. A dissipative system is called passive if $Y=U=\mathbb{R}^{m}, X=\mathbb{R}^{n}, V_{s}(0)=0$ and (14) holds for the particular supply rate $w(u, y)=u^{\mathrm{T}} y$. A passive system is called lossless if (14) holds with equality for all $t>0.8,9,13,14$

Here, we consider systems of the form

$$
\begin{aligned}
& \dot{x}(t)=f(x(t))+G(x(t)) u(t) \\
& y(t)=h(x(t))+J(x(t)) \phi(x(t), u(t), t) u(t)
\end{aligned}
$$

with internal state $x(t) \in \mathbb{R}^{n}$, input $u(t) \in \mathbb{R}^{m}$, output $y(t) \in \mathbb{R}^{m}$, and non-negative scalar function $\phi: \mathbb{R}^{n} \times \mathbb{R}^{m} \times[0, \infty) \rightarrow[0, \infty)$. We assume $f(0)=0, h(0)=0$, and $f(\cdot), G(\cdot), h(\cdot)$ and $J(\cdot)$ are smooth functions of appropriate dimension. Furthermore, the system (15), (16) is assumed to be zero-state detectable and completely reachable, where zero-state detectable means $u(t) \equiv 0$, $y(t) \equiv 0$ implies $x(t) \equiv 0$, and completely reachable means that for all states $x_{0}, x_{1}$ there exists a finite time $t_{1}$ and a square-integrable control $u(t)$ defined on $\left[0, t_{1}\right]$ such that the state can be driven from $x(0)=x_{0}$ to $x\left(t_{1}\right)=x_{1}$. In this paper, when a controller is represented in the form (15), (16), we will include the subscript 'c' and interchange the roles of $u$ and $y$. Note that due to the presence of the multiplier $\phi(16)$ is not affine in the control, and thus cannot be addressed by the framework in Reference 10. Passive systems that are not affine in the control are also considered in Reference 17.

The following result for systems of the form (15), (16) with $\phi(x, u, t) \equiv 1$ characterizes a passive system in terms of KYP-type conditions. 


\section{Lemma 1 (Reference 10)}

The system (15), (16) with $\phi(x, u, t) \equiv 1$ is passive if and only if there exist functions $V_{\mathrm{s}}: \mathbb{R}^{n} \rightarrow[0, \infty), l: \mathbb{R}^{n} \rightarrow \mathbb{R}^{p}$ and $W: \mathbb{R}^{n} \rightarrow \mathbb{R}^{p \times m}$ with $V_{\mathrm{s}}(x)$ continuously differentiable, $V_{\mathrm{s}}(0)=0$, such that, for all $x \in \mathbb{R}^{n}$,

$$
\begin{aligned}
V_{\mathrm{s}}^{\prime}(x) f(x) & =-l^{\mathrm{T}}(x) l(x) \\
\frac{1}{2} V_{\mathrm{s}}^{\prime}(x) G(x) & =h^{\mathrm{T}}(x)-l^{\mathrm{T}}(x) W(x) \\
J(x)+J^{\mathrm{T}}(x) & =W^{\mathrm{T}}(x) W(x)
\end{aligned}
$$

For systems that do not satisfy $\phi(x, u, t) \equiv 1$, the following result provides a sufficient condition for passivity.

\section{Lemma 2}

Consider the system (15), (16). Assume there exists a function $V_{\mathrm{s}}: \mathbb{R}^{n} \rightarrow[0, \infty)$ that is continuously differentiable, and $V_{\mathrm{s}}(0)=0$. If

$$
\begin{aligned}
V_{\mathrm{s}}^{\prime}(x) f(x) & \leqslant 0 \\
V_{\mathrm{s}}^{\prime}(x) G(x) & =h^{\mathrm{T}}(x) \\
J(x)+J^{\mathrm{T}}(x) & \geqslant 0
\end{aligned}
$$

then (15), (16) is passive.

Proof. Using (20)-(22) it follows that

$$
\begin{aligned}
\int_{0}^{T} u^{\mathrm{T}}(t) y(t) \mathrm{d} t & =\int_{0}^{T}\left[u^{\mathrm{T}}(t) h(x(t))+u^{\mathrm{T}}(t) J(x(t)) \phi(x(t), u(t), t) u(t)\right] \mathrm{d} t \\
& =\int_{0}^{T}\left[u^{\mathrm{T}}(t) h(x(t))+\phi(x(t), u(t), t) u^{\mathrm{T}}(t) J(x(t)) u(t)\right] \mathrm{d} t \\
& \geqslant \int_{0}^{T} h^{\mathrm{T}}(x(t)) u(t) \mathrm{d} t \\
& =\int_{0}^{T} V_{\mathrm{s}}^{\prime}(x(t)) G(x(t)) u(t) \mathrm{d} t \\
& =\int_{0}^{T}\left[V_{\mathrm{s}}^{\prime}(x(t))(f(x(t))+G(x(t)) u(t))-V_{\mathrm{s}}^{\prime}(x(t)) f(x(t))\right] \mathrm{d} t \\
& =\int_{0}^{T}\left[\frac{\mathrm{d}}{\mathrm{d} t} V_{\mathrm{s}}(x(t))-V_{\mathrm{s}}^{\prime}(x(t)) f(x(t))\right] \mathrm{d} t \\
& =V_{\mathrm{s}}(x(T))-V_{\mathrm{s}}(x(0))+\int_{0}^{T}-V_{\mathrm{s}}^{\prime}(x(t)) f(x(t)) \mathrm{d} t \\
& \geqslant V_{\mathrm{s}}(x(T))-V_{\mathrm{s}}(x(0))
\end{aligned}
$$

which verifies (14) with $w=u^{\mathrm{T}} y$. Hence (15), (16) is passive. 
Two well-known properties of passive systems will be exploited in this paper: passive systems are stable in the sense of Lyapunov, and the negative feedback interconnection of two passive systems is passive with respect to the inputs and outputs of either of the original systems, and thus is Lyapunov stable. ${ }^{11,12}$

\section{PASSIVE CONTROLLERS}

Our primary objective for controller design is to asymptotically stabilize the origin $x=0$ of the system (3). In order to exploit the stability robustness property associated with the feedback connection of a passive plant with a passive controller, we must first ensure that the plant to be controlled is passive. However, due to the rigid-body rotational mode, the undamped RTAC model (3) is actually unstable, and, accordingly, not passive. Therefore, the approach we take for control design is to passify the plant model using bounded state feedback, and then investigate the performance of various asymptotically stabilizing passive control designs.

Note that our stability objective, namely, asymptotic stability of the origin, necessarily redefines the equilibrium structure of the system, since the equilibria of the open-loop system have the form $q=0, \dot{q}=0$ and $\dot{\theta}=0$, with $\theta$ arbitrary. In our passive controller designs, we include the condition that the controller should not distinguish between the states $\theta \bmod 2 \pi$, since the inability of the controller to recognize this physical equivalence can result in undesirable unwinding behaviour. ${ }^{3}$ An example of unwinding is given in Section 6.2.

\subsection{Passifying the plant model}

To passify the plant model, let $g>0$ and set

$$
N=-m g e \sin \theta+u
$$

where the input $u$ will be determined later based on the output of a passive controller. This inner-loop control is chosen because it is a bounded function of the state, and because it will not cause unwinding. The term $-m g e \sin \theta$ in (23) lends itself to the interpretation of the gravitational torque that would result if the eccentric arm were in a gravitational field of strength $g$ oriented in the $\theta=0$ direction. Consequently, the inner-loop system can be viewed as the emulation of a pendulum absorber, which is used to reduce vibration levels in tower structures. ${ }^{18}$ Tuning of this emulated absorber can be accomplished by adjusting the value $g$.

With the control (23) and in the absence of a disturbance force $F$, (3) becomes

$$
\dot{x}=\bar{f}(x)+g(x) u
$$

where

$$
\bar{f}(x)=\left[\begin{array}{c}
\dot{q} \\
\frac{-\left(I+m e^{2}\right)\left(k q-m e \dot{\theta}^{2} \sin \theta\right)+m e \cos \theta m g e \sin \theta}{(M+m)\left(I+m e^{2}\right)-(m e \cos \theta)^{2}} \\
\dot{\theta} \\
\frac{m e \cos \theta\left(k q-m e \dot{\theta}^{2} \sin \theta\right)-(M+m) m g e \sin \theta}{(M+m)\left(I+m e^{2}\right)-(m e \cos \theta)^{2}}
\end{array}\right]
$$


Now, consider the storage function

$$
V_{\mathrm{s}}(x) \triangleq \frac{1}{2}(M+m) \dot{q}^{2}-m e \dot{q} \dot{\theta} \cos \theta+\frac{1}{2}\left(I+m e^{2}\right) \dot{\theta}^{2}+\frac{1}{2} k q^{2}+m g e(1-\cos \theta)
$$

which represents the kinetic energy of the cart and arm masses, the potential energy stored in the spring element, and the potential function associated with (23). Corresponding to (20), (21) we have

$$
\begin{aligned}
& V_{\mathrm{s}}^{\prime}(x) \bar{f}(x)=0 \\
& V_{\mathrm{s}}^{\prime}(x) g(x)=\dot{\theta}
\end{aligned}
$$

so that, according to Lemma 2, (24) with input $u$ and output

$$
y=\dot{\theta}
$$

is passive, and (24), (29) will be termed the passified plant.

\subsection{Damped pendulum absorber emulation}

In this subsection, we design a controller that asymptotically stabilizes the passified plant (24), (29). Let $\alpha, \gamma>0$, and

$$
u=-\alpha \tanh \gamma \dot{\theta}
$$

which has the desirable property of being a bounded function of $\dot{\theta}$. This controller effectively adds a bounded damping term to the pendulum absorber designed in the inner loop, and the resulting controller emulates a damped pendulum absorber. Note that the parameter $\alpha$ determines the saturation level for the controller, while the product $\alpha \gamma$ defines the small amplitude gain, since the linearization of $\alpha \tanh \gamma \dot{\theta}$ about $\dot{\theta}=0$ is $\alpha \gamma \dot{\theta}$. With the control input defined by (23) and (30), the control torque $N$ is bounded in magnitude by $m g e+\alpha$.

The closed-loop system (24), (29), (30) is given by

$$
\dot{x}=\bar{f}(x)-g(x) \alpha \tanh \gamma \dot{\theta}
$$

By choosing $V(x)=V_{\mathrm{s}}(x)$ to be a Lyapunov candidate, where $V_{\mathrm{s}}(x)$ is given in (26), along the trajectories of the closed-loop system we have

$$
\dot{V}(x)=-\alpha \dot{\theta} \tanh \gamma \dot{\theta} \leqslant 0
$$

and asymptotic stability of the origin follows from the invariant set theorem. ${ }^{19}$ It also follows from the invariant set theorem that the closed-loop trajectory from every initial condition will asymptotically approach an equilibrium position in state space of the form $q=0, \dot{q}=0, \dot{\theta}=0$, $\theta=n \pi, n=0, \pm 1, \pm 2, \ldots$, which corresponds to one of two physical configurations: a stable configuration corresponding to the arm pointed 'down' $(\theta=0 \bmod 2 \pi)$, or an unstable configuration corresponding to the arm pointed 'up' $(\theta=\pi \bmod 2 \pi)$. Furthermore, if a particular trajectory tends to asymptotically approach the unstable configuration, a small disturbance would cause it 


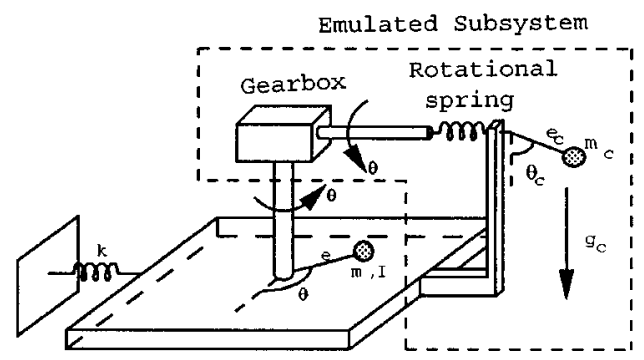

Figure 3. Coupled pendula absorber

to approach the stable configuration. While this is desirable global behavior, the origin $x=0$ is not globally asymptotically stable.

\subsection{Coupled pendula absorber emulation}

In this subsection we extend the emulated damped pendulum absorber design of Section 5.2 to emulate the multi-mode absorber comprised of a system of coupled pendula ${ }^{4}$ as shown in Figure 3. This added flexibility can be exploited by tuning the controller to effectively reject disturbances with two dominant frequency components, or to efficiently stabilize a multi-mode plant as in Reference 20. These capabilities are not explored further in this paper, however.

For the coupled pendulum absorber, the pendulum emulated by the eccentric arm and the damped pendulum control law (30) is termed the primary pendulum. The angular motion of the primary pendulum is imagined to be transmitted by a massless rigid rod and ideal gearbox assembly to an auxiliary pendulum. A nonlinear rotational spring element is introduced to couple the dynamics of the two pendula. The auxiliary pendulum hangs vertically in an emulated gravitational field of strength $g_{\mathrm{c}}$, and the damping is represented by $-d_{\mathrm{c}} \dot{\theta}_{\mathrm{c}}$. Note that since the rotational motion of the auxiliary pendulum is normal to the direction of motion of the cart, its physical realization does not directly produce a reaction force on the cart, but rather only produces a reaction force on the primary pendulum. Since the control input is torque applied to the eccentric arm, we are thus able to emulate the effect of this auxiliary pendulum subsystem.

The primary pendulum is defined by the parameters $m, I$ and $e$, the $\theta$ degree of freedom, and controller parameters $g, \alpha$ and $\gamma$ as before. The auxiliary pendulum is defined by a virtual mass $m_{\mathrm{c}}$ at a distance $e_{\mathrm{c}}$ about the rotational axis, and the $\theta_{\mathrm{c}}$ degree of freedom, measured from the vertical. The inertia of the auxiliary pendulum about the pivot is $I_{\mathrm{c}}$. The nonlinear rotational spring that couples the primary and auxiliary pendula has restoring torque $-\kappa \sin \left(\theta_{\mathrm{c}}-\theta\right)$, where $\kappa>0$.

To realize the coupled pendula absorber in Figure 3 as a passive compensator, we write the equations of motion with input $y=\dot{\theta}$ and output $-u$ as

$$
\begin{aligned}
\dot{x}_{\mathrm{c}} & =f_{\mathrm{c}}\left(x_{\mathrm{c}}\right)+G_{\mathrm{c}}\left(x_{\mathrm{c}}\right) y \\
-u & =h_{\mathrm{c}}\left(x_{\mathrm{c}}\right)+J_{\mathrm{c}}\left(x_{\mathrm{c}}\right) \phi\left(x_{\mathrm{c}}, y, t\right) y
\end{aligned}
$$


where $x_{\mathrm{c}}=\left[\begin{array}{lll}\theta & \theta_{\mathrm{c}} & \dot{\theta}_{\mathrm{c}}\end{array}\right]^{\mathrm{T}}$, and

$$
\begin{gathered}
f_{\mathrm{c}}\left(x_{\mathrm{c}}\right)=\left[\begin{array}{c}
0 \\
-\frac{\kappa \sin \left(\theta_{\mathrm{c}}-\theta\right)}{I_{\mathrm{c}}}-\frac{\dot{\theta}_{\mathrm{c}} g_{\mathrm{c}} e_{\mathrm{c}} \sin \theta_{\mathrm{c}}}{I_{\mathrm{c}}}-\frac{d_{\mathrm{c}}}{I_{\mathrm{c}}} \dot{\theta}_{\mathrm{c}}
\end{array}\right], \quad G_{\mathrm{c}}\left(x_{\mathrm{c}}\right)=\left[\begin{array}{l}
1 \\
0 \\
0
\end{array}\right] \\
h_{\mathrm{c}}\left(x_{\mathrm{c}}\right)=\kappa \sin \left(\theta-\theta_{\mathrm{c}}\right), \quad J_{\mathrm{c}}\left(x_{\mathrm{c}}\right)=\alpha, \quad \phi\left(x_{\mathrm{c}}, y, t\right)=\frac{\tanh \gamma y}{y}
\end{gathered}
$$

Notice that since $0<\phi(y)<1$, (33), (34) is of the form (15), (16).

A storage function for the compensator (33)-(36) is given by

$$
V_{\mathrm{sc}}\left(x_{\mathrm{c}}\right) \triangleq \frac{1}{2} I_{\mathrm{c}} \dot{\theta}_{\mathrm{c}}^{2}+m_{\mathrm{c}} g_{\mathrm{c}} e_{\mathrm{c}}\left(1-\cos \theta_{\mathrm{c}}\right)+\kappa\left(1-\cos \left(\theta-\theta_{\mathrm{c}}\right)\right)
$$

which represents the kinetic energy of the auxiliary pendulum plus the potential energies of the auxiliary pendulum and the elastic coupling. The system (33)-(36) with input $y$, output $-u$, and storage function $V_{\mathrm{sc}}\left(x_{\mathrm{c}}\right)$ satisfies

$$
\begin{gathered}
V_{\mathrm{sc}}^{\prime}\left(x_{\mathrm{c}}\right) f_{\mathrm{c}}\left(x_{\mathrm{c}}\right)=0 \\
V_{\mathrm{sc}}^{\prime}\left(x_{\mathrm{c}}\right) G_{\mathrm{c}}\left(x_{\mathrm{c}}\right)=h_{\mathrm{c}}^{\mathrm{T}}\left(x_{\mathrm{c}}\right) \\
J_{\mathrm{c}}\left(x_{\mathrm{c}}\right)+J_{\mathrm{c}}^{\mathrm{T}}\left(x_{\mathrm{c}}\right)=2 \alpha>0
\end{gathered}
$$

corresponding to the conditions of Lemma 2. The compensator is thus passive.

Because the closed-loop system is comprised of the negative feedback interconnection of passive systems, it is passive, and thus Lyapunov stable. Asymptotic stability of the closed-loop system follows from the invariant set theorem, where $V_{\mathrm{cl}}\left(x, x_{\mathrm{c}}\right)=V_{\mathrm{s}}(x)+V_{\mathrm{sc}}\left(x_{\mathrm{c}}\right)$ is used as a Lyapunov function for the closed-loop system. It also follows from the invariant set theorem that the stability properties of the equilibria $q=0, \dot{q}=0, \dot{\theta}=0, \dot{\theta}_{\mathrm{c}}=0, \theta= \pm n_{1} \pi, \theta_{\mathrm{c}}= \pm n_{2} \pi$ for integers $n_{1}$ and $n_{2}$, are qualitatively similar to those of the closed-loop system involving the damped pendulum absorber of Section 5.2 in that all closed-loop trajectories asymptotically approach the origin, modulo $\pi$ in the rotational states $\theta, \theta_{c}$.

\subsection{Virtual resetting absorber controllers}

The third dissipative controller we consider is a virtual resetting absorber controller, which emulates an absorber system whose states are reset to achieve instantaneous reduction of the 'total energy' of the closed-loop system, where the total energy includes the kinetic and potential energies associated with the actual physical plant, as well as the emulated energy associated with the states of the controller. One type of virtual resetting controller, called a virtual trap-door absorber, is described in Reference 15, where the resetting algorithm is used to achieve finite-time stabilization of the double integrator. A general theory of virtual resetting absorber controllers is developed in Reference 16. Resetting differential systems are also considered in References 21 and 22 as a special case of differential equations with impulse effect. 
Resetting differential systems consist of three main elements: a continuous-time dynamical equation, which governs the motion of the system between resetting events; a difference equation, which governs the way the states of the controller are instantaneously changed when a resetting event occurs; and a condition that determines when the states of the system are to be reset. The evolution of the state of a resetting differential system is as follows: while the resetting condition is not met, the state is given by the solution of the differential equation, with appropriate initial conditions. Upon reaching a point in time and/or state space that satisfies the resetting condition, the state of the system is instantaneously reset according to the resetting law. The state then proceeds to evolve as a solution of the differential equation again, until the resetting condition is again satisfied.

The resetting controller is given by

$$
\begin{aligned}
\dot{x}_{\mathrm{c}} & =\left[\begin{array}{c}
\dot{\theta}_{\mathrm{c}} \\
-\frac{\kappa}{I_{\mathrm{c}}} \sin \left(\theta_{\mathrm{c}}-\theta\right)
\end{array}\right], & \kappa \dot{\theta} \sin \left(\theta-\theta_{\mathrm{c}}\right)>0 \\
\Delta x_{\mathrm{c}} & =\left[\begin{array}{c}
\theta-\theta_{\mathrm{c}} \\
-\dot{\theta}_{\mathrm{c}}
\end{array}\right], & \kappa \dot{\theta} \sin \left(\theta-\theta_{\mathrm{c}}\right) \leqslant 0 \\
u & =\kappa \sin \left(\theta_{\mathrm{c}}-\theta\right) &
\end{aligned}
$$

The continuous-time equation governing the behavior of the state $x_{\mathrm{c}}$ between resetting events is given by (41). These dynamics represent the coupled pendula absorber of Section 5.3 where the gravity term $g_{\mathrm{c}}$ and the dissipation term $d_{\mathrm{c}}$ are both set to zero. Notice that unlike the passive controller designs of Sections 5.2 and 5.3, this controller has no dissipation term in its dynamics (41).

The resetting law (42) describes the instantaneous change in the controller state that occurs when the resetting condition is met; that is, when the resetting condition is met, the state $x_{\mathrm{c}}$ is instantly reset to $x_{\mathrm{c}}+\Delta x_{\mathrm{c}}$, and thus, according to (42), $\theta_{\mathrm{c}}$ is reset to $\theta_{\mathrm{c}}+\theta-\theta_{\mathrm{c}}=\theta$, and $\theta_{\mathrm{c}}$ is reset to $\dot{\theta}_{\mathrm{c}}-\dot{\theta}_{\mathrm{c}}=0$. This resetting law (42) thus resets $\theta_{\mathrm{c}}$ to $\theta$ and $\dot{\theta}_{\mathrm{c}}$ to 0 .

The resetting condition in (41), (42) involves a sign condition on the function $\kappa \dot{\theta} \sin \left(\theta-\theta_{\mathrm{c}}\right)$. The motivation for this condition, as well as for the form of the reset law (42), is based on properties of the emulated energy, which is given by

$$
V_{\mathrm{c}}\left(x_{\mathrm{c}}, x\right)=\frac{1}{2} I_{\mathrm{c}} \dot{\theta}_{\mathrm{c}}^{2}+\kappa\left(1-\cos \left(\theta-\theta_{\mathrm{c}}\right)\right) \geqslant 0
$$

When the resetting condition is not met, it follows from (41) that

$$
\frac{\mathrm{d}}{\mathrm{d} t} V_{\mathrm{c}}\left(x_{\mathrm{c}}, x\right)=\kappa \dot{\theta} \sin \left(\theta-\theta_{\mathrm{c}}\right)
$$

It follows from (24)-(28) and (41)-(45) that

$$
\frac{\mathrm{d}}{\mathrm{d} t} V_{\mathrm{c}}\left(x_{\mathrm{c}}, x\right)=\kappa \dot{\theta} \sin \left(\theta-\theta_{\mathrm{c}}\right)=-\dot{\theta} u=-\frac{\mathrm{d}}{\mathrm{d} t} V_{\mathrm{s}}(x)
$$

Thus, $V_{\mathrm{c}}$ is increasing if and only if $V_{\mathrm{s}}$ is decreasing. It is now clear from (41), (42) and (45) that the resetting condition (42) allows the states of the controller to evolve without resetting as long as 
the emulated energy (44) is increasing; that is, no resetting occurs provided the controller is removing energy from the plant (24). When the controller is no longer able to remove energy from the RTAC, the controller states are reset with $\theta_{\mathrm{c}}$ reset to $\theta$, and $\dot{\theta}_{\mathrm{c}}$ reset to 0 . This change in controller state causes the emulated energy (44) to be instantly transferred from $V_{\mathrm{c}}\left(x_{\mathrm{c}}, x\right) \geqslant 0$ to $V_{\mathrm{c}}\left(x_{\mathrm{c}}+\Delta x_{\mathrm{c}}, x\right)=0$, effectively dumping any 'energy' that had accumulated in the controller.

In this approach, energy is allowed to flow from the plant into the controller, but due to the resetting mechanism, no energy can flow from the controller back to the plant. Hence, in the discussion that follows, we will refer to this resetting controller as the virtual one-way absorber, or simply the one-way absorber. While this resetting algorithm has no mechanism for doing positive work on the plant, its discrete resetting action (42) prevents it from being captured by the standard dissipativity framework.

To implement the one-way absorber controller on the RTAC, only the angle $\theta$ is measured. The value of the compensator energy (44) is evaluated at each time step, and the controller states are reset whenever the compensator energy is less than or equal to the value of the compensator energy at the previous sampling instant.

\section{EXPERIMENTAL RESULTS}

\subsection{Performance comparison}

In this section, the controller designs of Sections 3 and 5 are implemented and evaluated on the RTAC testbed. The baseline experiment used to evaluate the performance of the controllers is an initial condition response, where $q(0)=1 \cdot 5^{\prime \prime}$ and $\dot{q}(0)=0$. This initial condition is roughly as large as the testbed will allow, and the responses are representative of those obtained at other initial conditions. For the passivity-based controllers, the arm is set initially to $\theta=0$ and $\dot{\theta}(0)=0$. With the RTAC held in this initial configuration, the controller is active, although no control torque is applied by the motor. The experiment begins when the cart is released. For the integrator backstepping controller, when the cart is held motionless at $q(0)=1 \cdot 5^{\prime \prime}$, control torques cause the arm to be initially at rest at approximately $\theta=145^{\circ}$.

The time histories of the cart position and arm angle are recorded, and the value of the control signal is computed by multiplying the output of the controller (measured in volts) times the transconductance of the servo drive $(0.53 \mathrm{~A} / \mathrm{V})$ times the motor constant $(3.04 \mathrm{oz}$ in/A). For each experiment, a settling time is computed as the time required for the cart displacement to become less than $10 \%$ of the initial value, that is, $0 \cdot 15^{\prime \prime}$. An approximate damping ratio is assigned to each response based on logarithmic decrement analysis. Clearly, the responses of these nonlinear systems are not expected to mimic the response of a linear system. However, by approximating the rate of decrease of the amplitude of the cart displacement during roughly the first $5 \mathrm{~s}$ of the experiment, an effective damping ratio can be assigned that is useful for comparison purposes. A summary of the performance of the various controllers is given in Table II.

The open-loop response to the given initial condition is given in Figure 4. Note that in the absence of control, the eccentric arm tends to drift into a position parallel with the axis of translation. In this case, the arm tends toward $-90^{\circ}$.

The integrator backstepping controller described in Section 3 is implemented with $p_{0}=5000$, $p_{1}=500, p_{2}=500, c_{0}=0 \cdot 5, c_{1}=1$ and $c_{2}=1$. These parameters were selected by trial and error as it is not clear precisely what role is played by the individual controller parameters in determining the closed-loop response. The strategy is to choose controller parameters that 
Table II. Summary of controller performance

\begin{tabular}{llc}
\hline Controller & $\zeta_{\text {approx }}$ & $u_{\max }$ \\
\hline Open loop & $0 \cdot 44 \%$ & - \\
Coupled pendula absorber & $3 \cdot 2 \%$ & $0 \cdot 52 \mathrm{oz} \mathrm{in}$ \\
Integrator backstepping & $3 \cdot 7 \%$ & $12 \cdot 2 \mathrm{oz} \mathrm{in}$ \\
& & (saturated) \\
Damped pendulum absorber & $5 \cdot 1 \%$ & $0 \cdot 47 \mathrm{oz} \mathrm{in}$ \\
Virtual resetting absorber & $5 \cdot 2 \%$ & $0 \cdot 44 \mathrm{ozin}$ \\
\hline
\end{tabular}
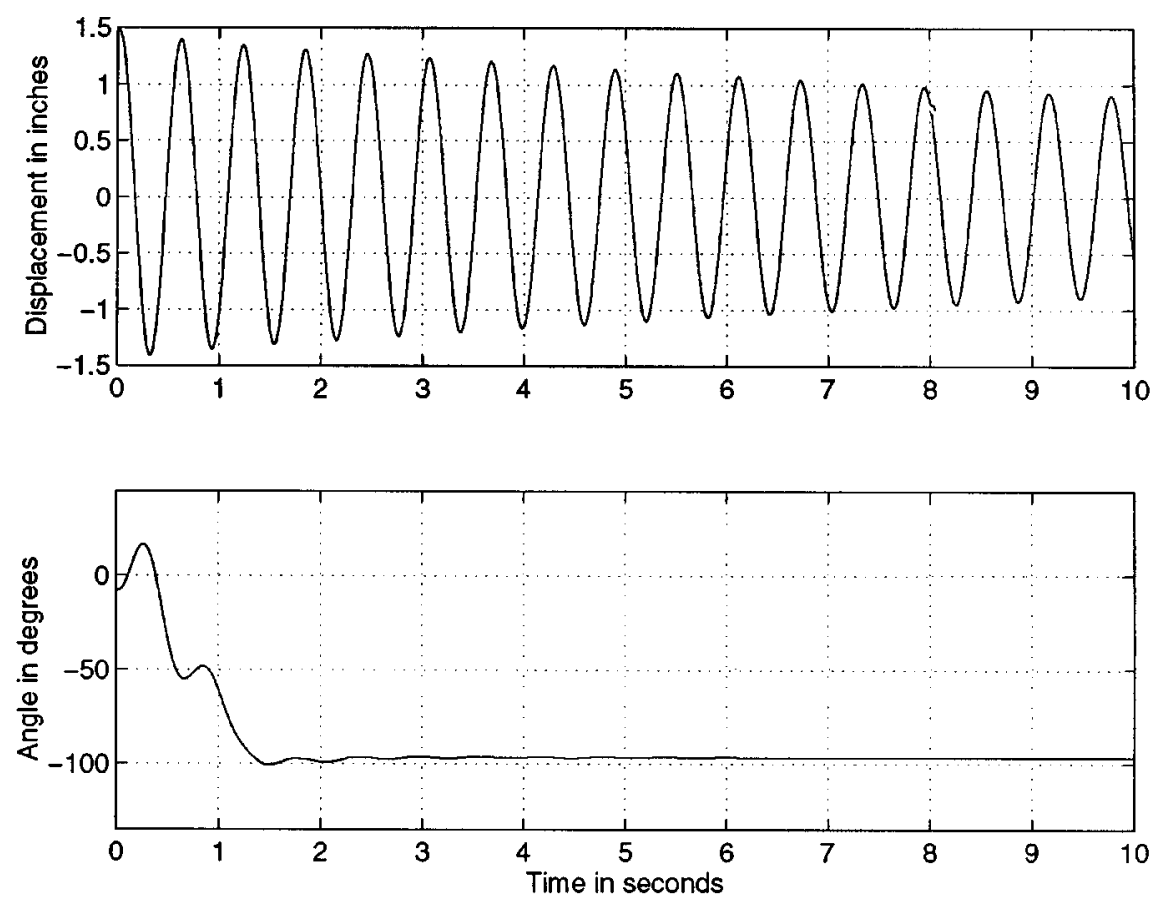

Figure 4. Uncontrolled response of the RTAC to a 1.5 in initial displacement

minimize the settling time from the given baseline initial condition. The factor most responsible for limiting the performance of this controller is the maximum current constraint. The current limiter is set to $4 \mathrm{~A}$ or $12 \cdot 2 \mathrm{oz}$ in of torque.

The experimental initial-condition response is shown in Figure 5. The initial angle of the arm is about $145^{\circ}$, which is due to the control torques generated by the full-state feedback controller when the cart is held at $q=1 \cdot 5^{\prime \prime}$. The integrator backstepping controller settles the cart to $10 \%$ of the initial displacement in about $6 \mathrm{~s}$. Based on logarithmic decrement analysis during this period, the effective damping ratio is approximately $3.7 \%$. The controller then continues to bring the cart and arm to rest at the desired zero position. The controller output is characterized by a large amplitude control signal — saturating often during the initial two seconds — with substantial high 

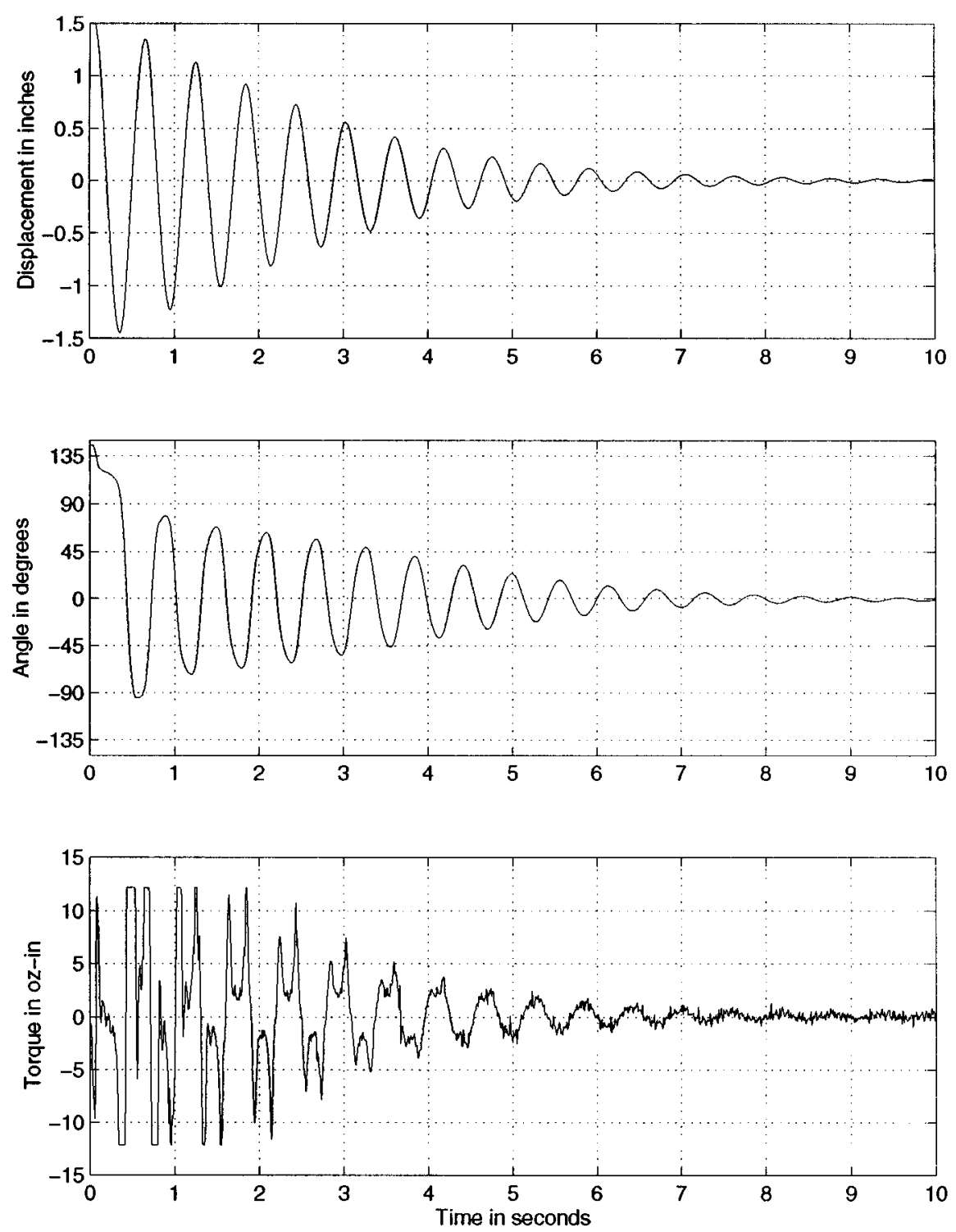

Figure 5. Integrator backstepping controller: response of the RTAC to a 1.5 in initial displacement

frequency content. It is interesting to note that prior to saturation at $12.2 \mathrm{oz}$ in, the commanded control torque was as large as $65 \mathrm{oz}$ in.

The damped pendulum absorber described in Section 5.2 is designed with $m=3.4 \mathrm{oz}$, $g=19.7 \mathrm{ft} / \mathrm{s}^{2}, e=2.33 \mathrm{in}, \alpha=10 \mathrm{oz}$ in and $\gamma=0.0057 \mathrm{~s}$. The parameters $m$ and $e$ are chosen to approximately reflect the actual mass and eccentricity of the arm. The parameter $g$ is tuned so that, in the absence of damping, the initial condition response of the cart excites the greatest amount of motion in the arm. This procedure helps tune the natural frequency of the absorber to 

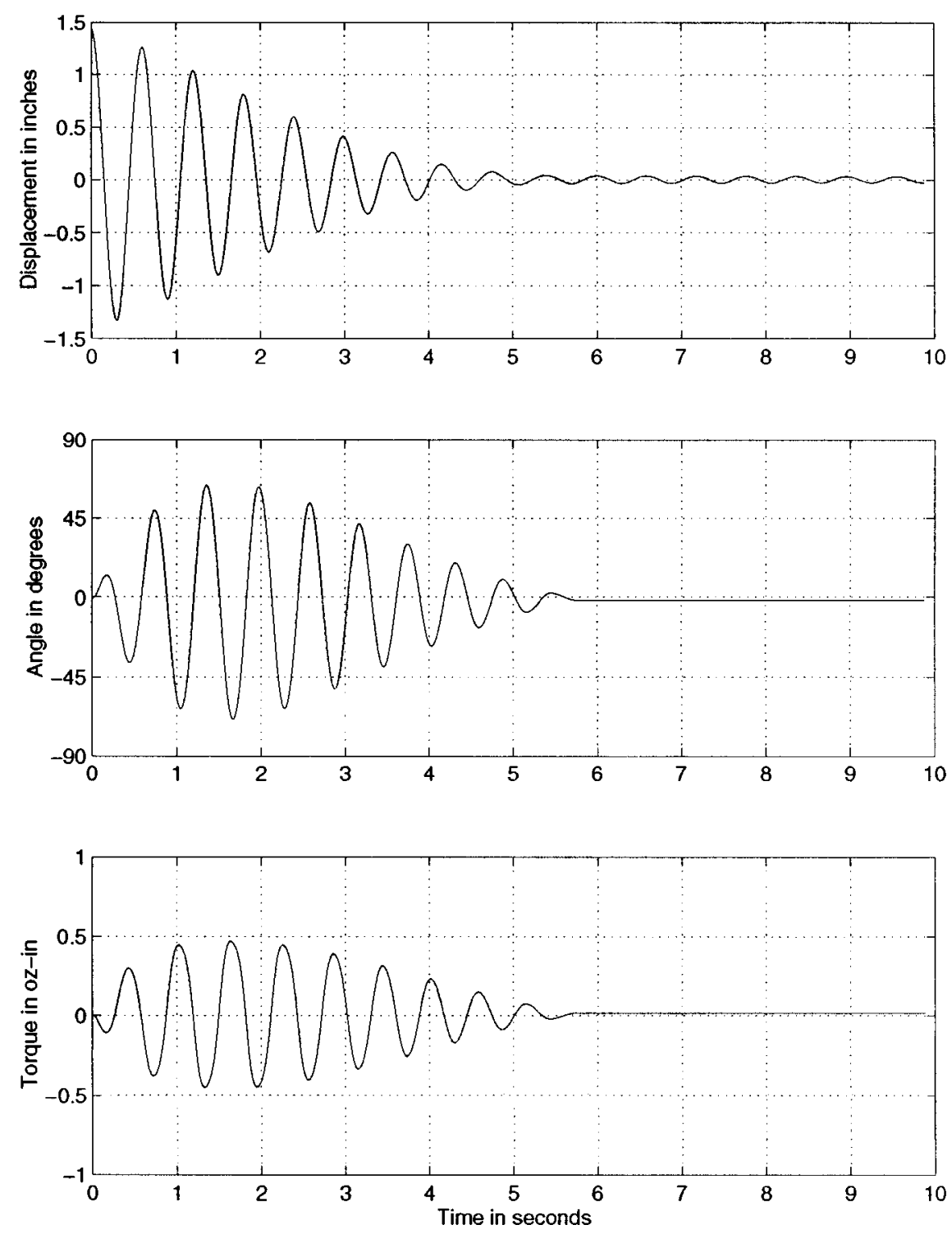

Figure 6. Damped pendulum absorber controller: response of the RTAC to 1.5 in initial displacement

the natural frequency of the cart, which is useful for efficiently transferring the energy from the cart to the arm where it can be dissipated. The parameter $\alpha$ is chosen first to set the torque bound, while the damping parameter $\gamma$ is tuned to provide adequate damping. If $\gamma$ is too large then the energy transfer from the cart to the arm becomes greatly reduced, whereas if $\gamma$ is too small then energy is transferred but not dissipated.

The damped pendulum absorber response to the baseline initial condition is given in Figure 6. The cart settling time is approximately $4.5 \mathrm{~s}$, corresponding to an approximate damping ratio 
of $5 \cdot 1 \%$. After this time, the cart suffers residual oscillations of approximately $0 \cdot 04^{\prime \prime}$ amplitude that are not damped by the controller. The control signal appears smooth.

The coupled pendula absorber described in Section 5.3 is designed with $m=3.4 \mathrm{oz}$, $g=34.6 \mathrm{ft} / \mathrm{s}^{2}, e=2.33 \mathrm{in}, \alpha=10 \mathrm{ozin}, \gamma=0.0057 \mathrm{~s}, \kappa=14.2 \mathrm{ozin}, I_{\mathrm{c}}=11.3 \mathrm{oz} \mathrm{in}^{2}, e_{\mathrm{c}}=0$, and $d_{\mathrm{c}}=14 \cdot 2 \mathrm{oz}$ in sec. Here again the parameters $m$ and $e$ are chosen to reflect the actual mass and eccentricity of the arm. The design of the remaining parameters is more complicated than the design of the damped pendulum absorber since the absorber subsystem here is of higher order. To achieve good settling behaviour, the parameters are tuned so that the initial condition response of the cart excites one 'mode' of the coupled pendulum absorber system as much as possible. The other 'mode' of the absorber could in theory be tuned to achieve improved disturbance rejection for disturbances at some other frequency, or to improve settling behaviour of some other mode of the plant.

The coupled pendula absorber response to the baseline initial condition is given in Figure 7. The cart settling time is approximately $6.75 \mathrm{~s}$, corresponding to an approximate damping ratio of $3 \cdot 2 \%$, based on logarithmic decrement analysis during the $5 \cdot 5 \mathrm{~s}$ of the experiment. The cart suffers residual oscillations of approximately $0 \cdot 1^{\prime \prime}$ amplitude that are not damped by the controller. The control signal appears smooth and appears to possess two dominant frequencies components.

The one-way absorber controller described in Section 5.4 is designed with $m=3.4 \mathrm{oz}$, $g=19.7 \mathrm{ft} / \mathrm{s}^{2}, e=2.33 \mathrm{in}, \kappa=4.25 \mathrm{oz}$ in and $I_{\mathrm{c}}=1.1 \mathrm{ozin}^{2}$. As was the case for the previous absorber-based controllers, the parameters $m$ and $e$ are chosen to reflect the actual mass and eccentricity of the arm. As it was for the damped pendulum absorber controller, the parameter $g$ is chosen to allow the most efficient energy transfer from the cart to the eccentric arm. Now, instead of proceeding to the selection of the proper dashpot parameter as we did in the damped pendulum absorber design, we simply design the controller parameters $\kappa$ and $I_{\mathrm{c}}$ to exchange energy efficiently with the eccentric arm. Since the one-way controller is implemented on a digital control board with a $200 \mu$ s sampling period, a comparison of the current value of the emulated energy (44) with the value of the emulated energy computed during the previous sample interval is used to determine whether or not the controller emulated energy has stopped increasing. When the emulated energy has stopped increasing, the states of the controller are reset so that the value of the controller energy function is zero.

The response of the one-way absorber controller to the baseline initial condition is given in Figure 8 . The settling time for this controller is approximately $4.5 \mathrm{~s}$ which corresponds to an approximate damping ratio of $5 \cdot 2 \%$. Residual cart oscillations of $0 \cdot 08^{\prime \prime}$ amplitude are not damped by the controller. Some discontinuity in the control signal due to the resetting nature of this controller is apparent in the figure.

One major difference between the integrator backstepping controller and the passive designs can be seen in the cart displacement response as time progresses. It has been noted in all of the passivity-based control cases that there is a residual oscillation of the cart that the controllers cannot remove. This is due to the stiction in the motor/arm assembly which is not included in the dynamical model and which causes the zero-state detectability condition to fail. For oscillations of this level, the accelerations of the cart are so small that the stiction force alone keeps the arm from moving, and, without motion in the arm, energy cannot be removed from the cart. To contrast, the integrator backstepping controller, being a full-state-feedback control law, applies torque based on measured cart displacement and velocity, and thus, despite the presence of stiction, can remove the low-amplitude cart oscillations that the passive controllers cannot. 

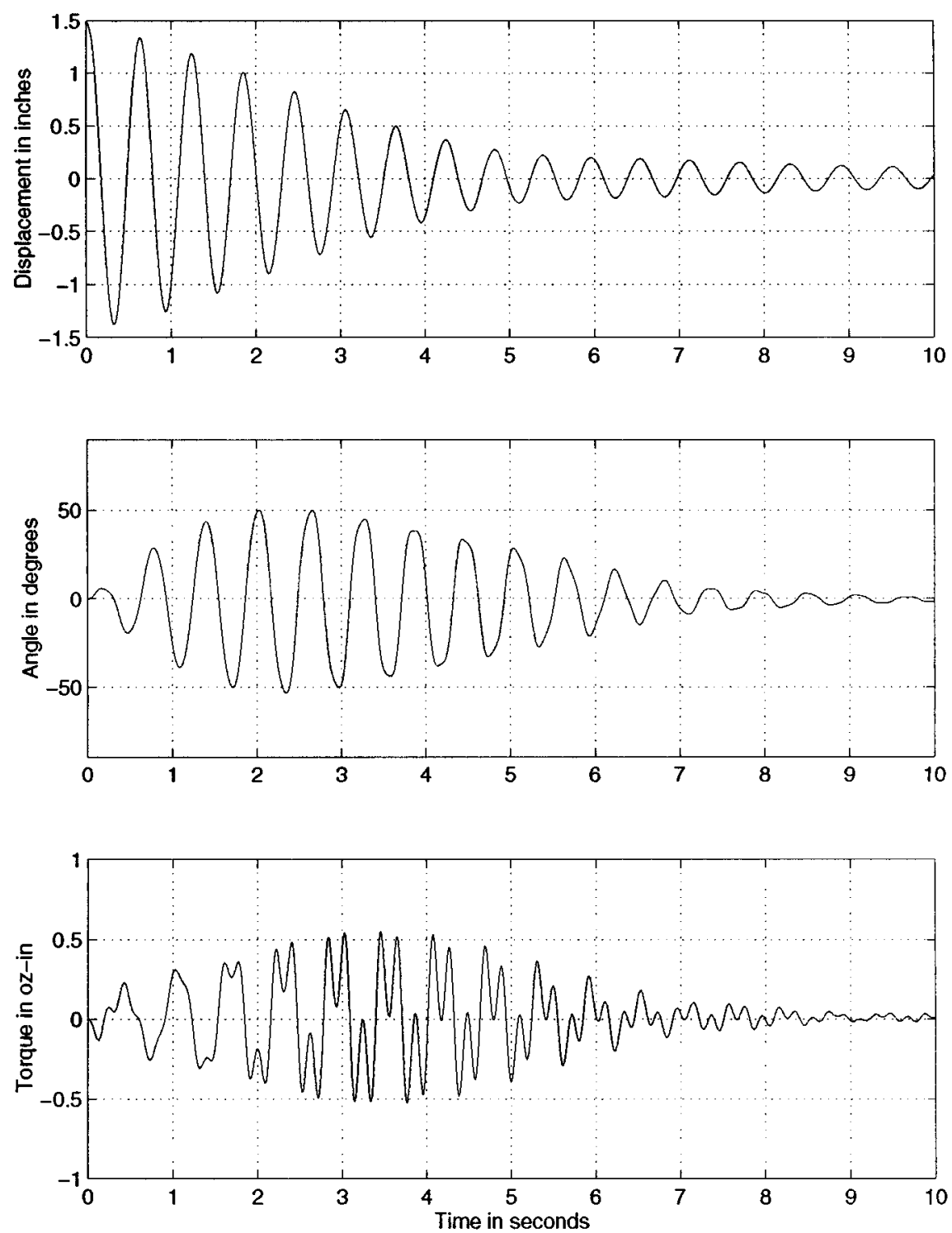

Figure 7. Coupled pendula absorber controller: response of the RTAC to a 1.5 in initial displacement

While all of the controllers achieve settling performance corresponding to an effective damping ratio in the $3-5 \%$ range, the nature of the control signals varies greatly. The control signal for the integrator backstepping controller can be seen to saturate often during the first two seconds of the experiment, and it has substantial high frequency content. In contrast, the control signals for the absorber-based controllers are characterized by relatively low amplitude, generally less than $0.5 \mathrm{oz}$ in, or about $4 \%$ of the level of the integrator backstepping controller. These control signals 

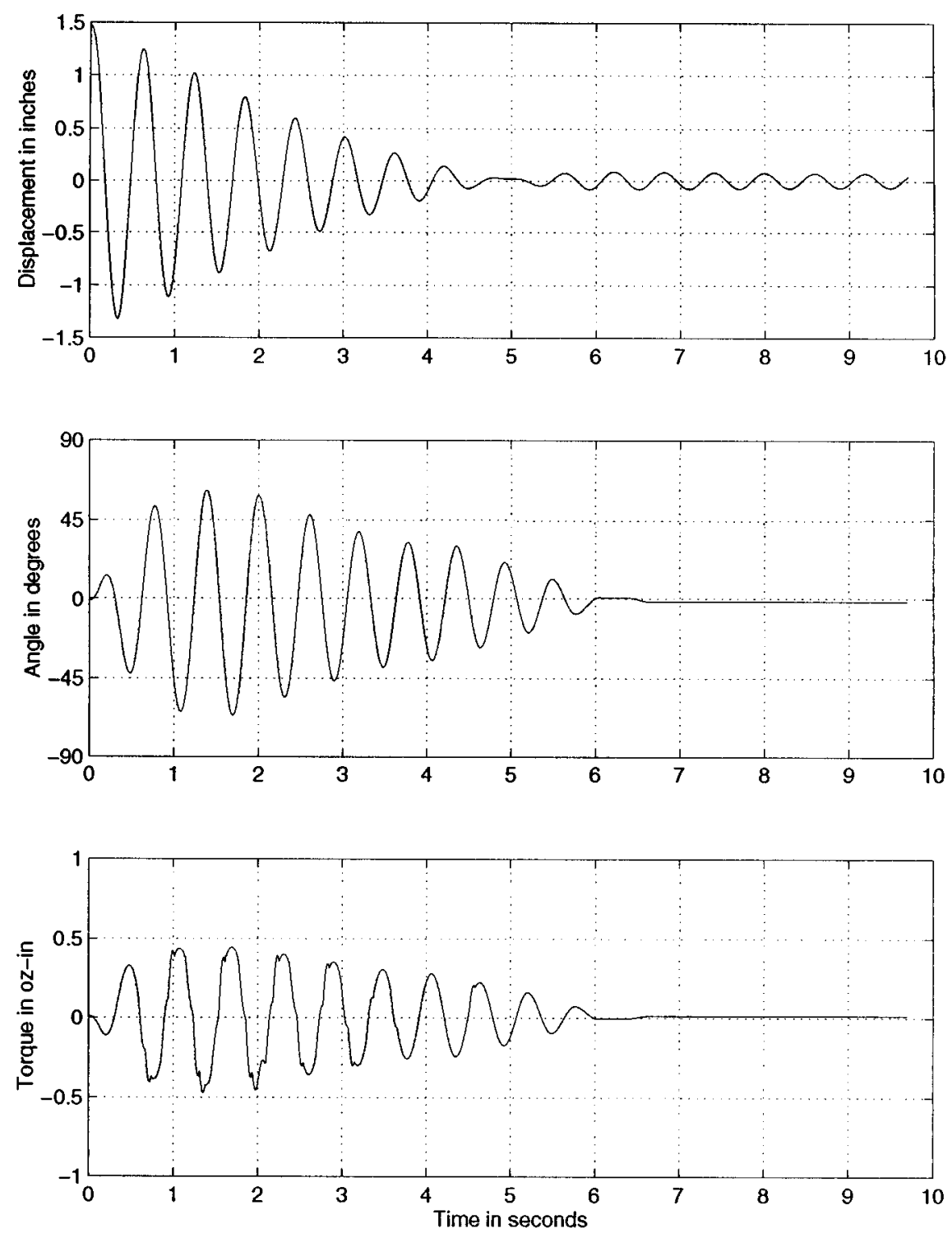

Figure 8. Virtual one-way absorber controller: response of the RTAC to a 1.5 in initial displacement

tend to be smooth in comparison with the backstepping controller, although small discontinuities due to controller resetting can be seen in the control signal for the virtual one-way absorber controller.

It is to be expected that the integrator backstepping controller could achieve better performance than the absorber-based controllers, since the former was not restricted by passivity constraints. In fact, simulations indicate that the integrator backstepping controller can achieve 

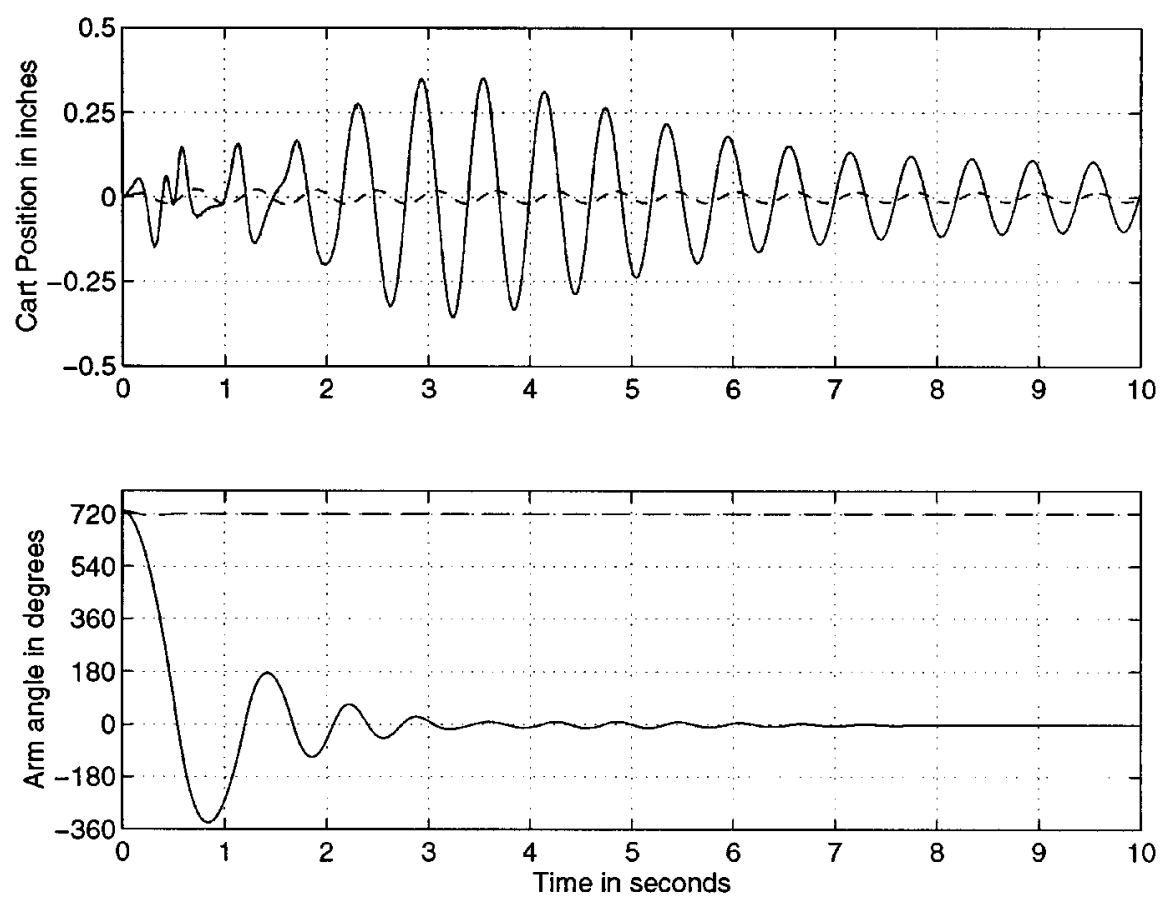

Figure 9. An example of controller unwinding: the globally stabilizing controller (solid line) unwinds from an initial angle of $730^{\circ}$ to zero, while the damped pendulum absorber controller (dashed line) converges to an angle of $720^{\circ}$

much lower settling times compared to passive designs. However, due to the control amplitude constraint imposed to safeguard the actuator, these ultra-high authority integrator backstepping controllers could not be implemented, and, although as implemented they used 25 times more control torque, the integrator backstepping controller was only able to achieve settling behaviour roughly comparable to the absorber-based controllers.

\subsection{Experimental example of unwinding}

To illustrate controller unwinding, we compare the response of the damped pendulum absorber controller with that of a globally asymptotically stabilizing controller of the form

$$
N=-m g e \tanh \theta-\alpha \gamma \dot{\theta}
$$

Unwinding of the integrator backstepping controller could not be demonstrated experimentally because of the excessive control signal amplitudes. Nonetheless, the unwinding behaviour of the controller (47) typifies the behaviour of controllers that globally asymptotically stabilize the origin $x=0$ of the RTAC.

The parameters $m, g, e, \alpha$ and $\gamma$ in (47) are chosen to be the same as for the damped pendulum absorber controller, and are given in Section 6.1. With these parameters, the damped pendulum absorber controller and the globally stabilizing controller (47) give very similar performance for the baseline initial condition. 
To illustrate unwinding, we consider the initial condition $q=0, \dot{q}=0, \dot{\theta}=0, \theta=730^{\circ}$, which represents the cart at rest at the zero position, and the arm at rest at two rotations plus $10^{\circ}$. The response of the RTAC is illustrated in Figure 9. The damped pendulum absorber controller causes the eccentric arm to move only $10^{\circ}$ to the $720^{\circ}$ position, exciting very little motion in the cart. In contrast, the globally stabilizing controller (47) does not recognize the $\theta=720^{\circ}$ and $\theta=0^{\circ}$ positions as equivalent, and therefore unwinds the arm to $0^{\circ}$. This unwinding creates large control signals, high rotational speeds for the arm, excites substantial motion in the cart, and overshoots the zero position by nearly a full revolution before coming to rest at the desired equilibrium position.

\section{CONCLUSIONS}

The RTAC experimental testbed was fully described. An integrator backstepping controller and three passive nonlinear controllers were designed and implemented on the RTAC, and the performance of the controllers in settling the RTAC system from a specified initial condition was evaluated and compared. In order to implement passive controllers, we first utilized an 'innerloop' control to passify the RTAC model. Three absorber-based, passive controllers were then considered: the damped pendulum absorber, which caused the arm to emulate a nonlinear, single-degree-of-freedom absorber; the coupled pendula abosrber, which caused the arm to emulate a nonlinear, multi-degree-of-freedom absorber; and the virtual one-way absorber, a type of virtual resetting lossless absorber controller that allows energy to flow into the virtual subsystem, but uses a resetting mechanism to ensure that the energy cannot return to the plant.

A disadvantage of the passive controllers is that they all suffer performance degradation due to the unmodelled stiction in the motor. The stiction causes the arm to remain at rest even though the cart undergoes small amplitude, very lightly damped oscillations. The integrator backstepping controller, being a full-state feedback controller, is able to use measurements of the cart position and velocity to generate control torques that overcome the stiction and bring the RTAC asymptotically to rest.

While all of the controllers considered achieved an effective damping ratio of $3-5 \%$ for the baseline initial condition considered, the integrator backstepping controller utilized 25 times more torque - saturating at $12.2 \mathrm{oz}$ in, compared to less than $0.5 \mathrm{oz}$ in for the passive controllers. While the passive controllers do not reach the maximum torque constraint, they also cannot make use of the extra available torque. The best performance of these absorber-based controllers is achieved by careful tuning of the control parameters, and 'increasing the gain' only serves to detune the controller. A controller that more efficiently uses the available torque to improve the settling performance of the RTAC would be desirable.

\section{ACKNOWLEDGEMENTS}

The research of R. T. Bupp and D. S. Bernstein was supported in part by the Air Force Office of Scientific Research under Grants F49620-93-1-0502 and F49620-95-1-0019 and the National Science Foundation under grant 9310735. The research of V. T. Coppola was supported in part by the National Science Foundation under Grant MS-9309165.

\section{REFERENCES}

1. Wan, C.-J., D. S. Bernstein and V. T. Coppola, 'Global stabilization of the oscillating eccentric rotor', in Proc. IEEE Conf. Dec. Contr., Orlando, FL, December 1994, pp. 4024-4029. 
2. Wan, C.-J., D. S. Bernstein and V. T. Coppola, 'Global stabilization of the oscillating eccentric rotor', Int. J. Nonlinear Mech., 10, 49-62 (1996).

3. Bupp, R. T., C.-J. Wan, V. T. Coppola and D. S. Bernstein, 'Design of a rotational actuator for global stabilization of translational motion', in Proc. ASME Winter Meeting, DE-Vol. 75, Chicago, IL, November 1994, pp. 449-456.

4. Bupp, R. T., D. S. Bernstein and V. T. Coppola, 'A benchmark problem for nonlinear control design: problem statement, experimental testbed, and passive nonlinear compensation', in Proc. Amer. Contr. Conf., Seattle, WA, June 1995, pp. 4363-4367.

5. Jankovic, M., D. Fontaine and P. V. Kokotović, 'TORA example: cascade- and passivity-based control designs', IEEE Control System Tech., 4, 292-297 (1996).

6. Marino, R., 'On the largest feedback linearizable subsystem', Systems Control Lett., 6, 345-351 (1986).

7. Kanellakopoulos, I., P. V. Kokotović and A. S. Morse, 'A toolkit for nonlinear feedback design', Systems Control Lett., 18, 83-92 (1992).

8. Willems, J. C., 'Dissipative dynamical systems, Part I: general theory', Arch. Rational Mech. Anal., 45, 321-351 (1972).

9. Willems, J. C., 'Dissipative dynamical systems, Part II: linear systems with quadratic supply rates', Arch. Rational Mech. Anal., 45, 352-393 (1972).

10. Moylan, P. J., 'Implications of passivity in a class of nonlinear systems', IEEE Trans. Automat. Control, 19, 373-381 (1974).

11. Hill, D. and P. J. Moylan, 'The stability of nonlinear dissipative systems', IEEE Trans. Automat. Control, 21, 708-711 (1976).

12. Hill, D. J. and P. J. Moylan, 'Stability results for nonlinear feedback systems', Automatica, 13, 377-382 (1977).

13. Hill, D. J. and P. J. Moylan, 'Dissipative dynamical systems: basic input-output and state properties', J. Franklin Inst., 309, 327-357 (1980).

14. Byrnes, C. I., A. Isidori and J. C. Willems, 'Passivity, feedback equivalence, and the global stabilization of minimum phase nonlinear systems', IEEE Trans. Automat. Control, 36, 1228-1240 (1991).

15. Bupp, R. T., D. S. Bernstein, V.-S. Chellaboina and W. M. Haddad, 'Finite-time stabilization of the double integrator using a virtual trap-door absorber', Proc. Conf. Contr. Appl., pp. 179-184, Dearborn, MI, September 1996.

16. Bupp, R. T., D. S. Bernstein, V.-S. Chellaboina and W. M. Haddad, 'Virtual resetting absorber controllers: theory and applications', Proc. Amer. Contr. Conf., pp. 2647-2651, Albuquerque, NM, June 1997.

17. Bernstein, D. S. and W. M. Haddad, 'Nonlinear controllers for positive real systems with arbitrary input nonlinearities', IEEE Trans. Automat. Control, 39, 1513-1517 (1994).

18. Korenov, B. G. and L. M. Reznikov, Dynamic Vibration Absorbers: Theory and Technical Applications, Wiley, New York, 1993.

19. Vidyasagar, M., Nonlinear Systems Analysis, Prentice-Hall, Englewood Cliffs, NJ, 1978.

20. Bupp, R. T., V. T. Coppola and D. S. Bernstein, 'Vibration suppression of multi-modal translational motion using a rotational actuator', in Proc. IEEE Conf. Dec. Control, Orlando, FL, December 1994, pp. 4030-4034.

21. Lakshmikantham, V., D. D. Bainov and P. S. Simeonov. Theory of Impulsive Differential Equations, Series in Modern Applied Mathematics, Vol. 6, World Scientific, Singapore, 1989.

22. Bainov, D. D. and P. S. Simeonov. Systems with Impulse Effect. Ellis Horwood Series in Mathematics and its Applications, Ellis Horwood, Chichester, U.K., 1989. 